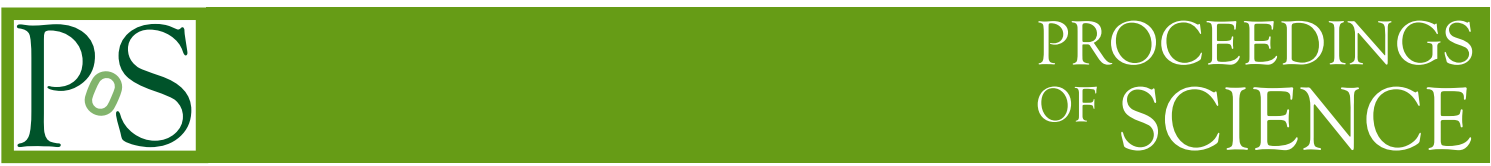

\title{
DBBC3 - the new wide-band backend for VLBI
}

\author{
G. Tuccari ${ }^{a}$, W. Alef ${ }^{b}$, S. Dornbusch ${ }^{b}$, M. Wunderlich ${ }^{b}$, A. Roy ${ }^{b}$, H. Rottmann ${ }^{b}$, \\ J. Wagner ${ }^{b}$, R. Haas ${ }^{c}$, K-Å.L. Johansson ${ }^{c}$ \\ a INAF Istituto di Radioastronomia, Sezione di Noto \\ Contrada Renna, 96017 Noto (SR), Italy \\ ${ }^{b}$ Max Planck Institute for Radio Astronomy \\ Auf dem Hügel 69, D-53121 Bonn, Germany \\ ${ }^{c}$ Onsala Space Observatory \\ SE-439 92 Onsala, Sweden \\ E-mail: walef@mpifr-bonn.mpg.de
}

The DBBC3 VLBI digital backend is the successor of the most widely adapted digital VLBI backend DBBC2. The DBBC3 offers much wider bandwidth and integrated Ethernet output. Three different firmwares for observing have been implemented until now: Direct Sampling Conversion (DSC), arbitrary selection of bands (OCT), and Digital Down Conversion (DDC). These modes cover all the requirements of the astronomical, VGOS and legacy geodetic VLBI of today, but also of the near future. In addition the DBBC3 offers unsurpassed compatibility to the relatively large number of other existing VLBI backends. A number of DBBC3 systems have been deployed and more are currently under construction, with the number of $4 \mathrm{GHz}$ bands ranging from 2 up to 8 with resulting typical output data-rates from $32 \mathrm{Gbps}$ to $128 \mathrm{Gbps}$. Laboratory and field tests have been performed.

14th European VLBI Network Symposium \& Users Meeting (EVN 2018)

8-11 October 2018

Granada, Spain 


\section{Introduction}

The DBBC3 has been developed with support by RadioNet3 in the Joint Research Activity DIVA $^{1}$ with the partners: INAF, MPIfR, OSO ${ }^{2}$. The DBBC3 is a VLBI backend for astronomy where it is or can be used for instance for EVN wide-band VLBI, the Global mm-VLBI Array (GMVA), and the Event Horizon Telescope (EHT). It is also a VLBI backend suitable for carrying out geodetic VGOS and legacy S/X-observations.

The DBBC3 is a further development of the DBBC2. It is backward compatible with the DBBC2 with respect to observing modes, control, and even some of the hardware. It offers from $1 \mathrm{IFs}$ to $8 \mathrm{IFs}$ on input with a maximum of $16 \mathrm{Gbps}$ to $128 \mathrm{Gbps}$ on output using 2-bit samples, as is the present standard in VLBI. With different firmware the theoretical maximum data-rate is $256 \mathrm{Gbps}$ for 2-bit data and up to $512 \mathrm{Gbps}$ for 8-bit samples.

The DBBC3 is now in a production status as the hardware has been finalised. 14 systems are operational. Most of them are for VGOS, two for the EHT, and three for EVN/GMVA stations. Two systems are under construction. The firmware development is much advanced (see details in section 3). A large effort has been invested to establish compatibility with all other existing VLBI backends, which for most cases has been achieved.

A number of test observations have been conducted in the last months to achieve the best performance for the VGOS observing modes and the EHT. Similar tests are planned for the EVN network and the GMVA.

\section{Hardware components}

The analogue conditioning module (GCoMo; see figure 1) has been superseded by GCoMo2. It has improved bandwidth response over $4 \mathrm{GHz}$, which has been achieved by replacing the $4 \mathrm{GHz}$ amplifiers by $6 \mathrm{GHz}$ ones. The GCoMo2 matches the receiver to the sampler module (ADB3L) with a lot of flexibility. The GCoMo2 takes pre-filtered signals up to $4 \mathrm{GHz}$ wide in the range $0 \mathrm{GHz}$ to $4 \mathrm{GHz}$. If the signal is not in the first Nyquist zone $(0 \mathrm{GHz}-4 \mathrm{GHz})$, on the GCoMo2

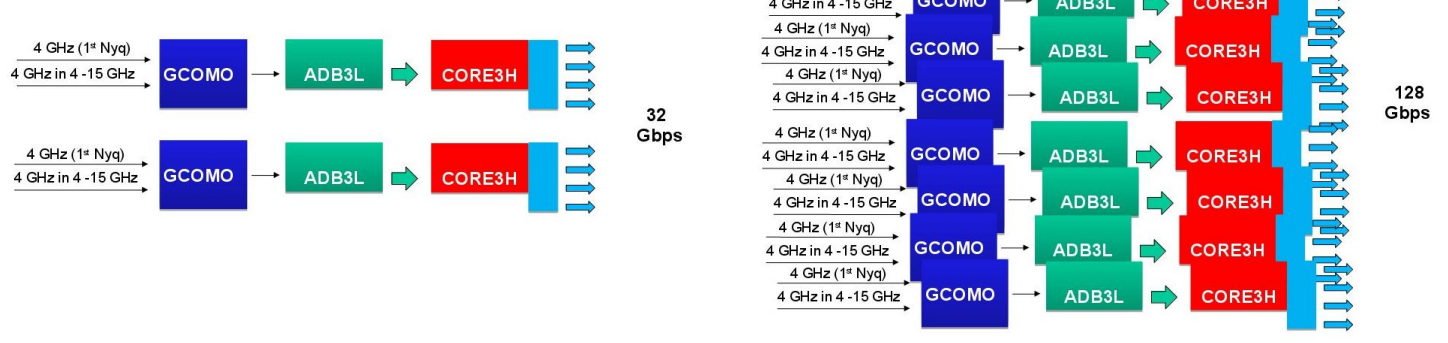

Figure 1: Left: block diagram for a DBBC3 with only 2 IFs. Right: Block diagram with 8 IFs.

\footnotetext{
${ }^{1}$ RadioNet3 has received funding from the European Commission Seventh Framework Programme (FP/20072013) under grant agreement No 283393.

${ }^{2}$ See list of author affiliations at top.
} 


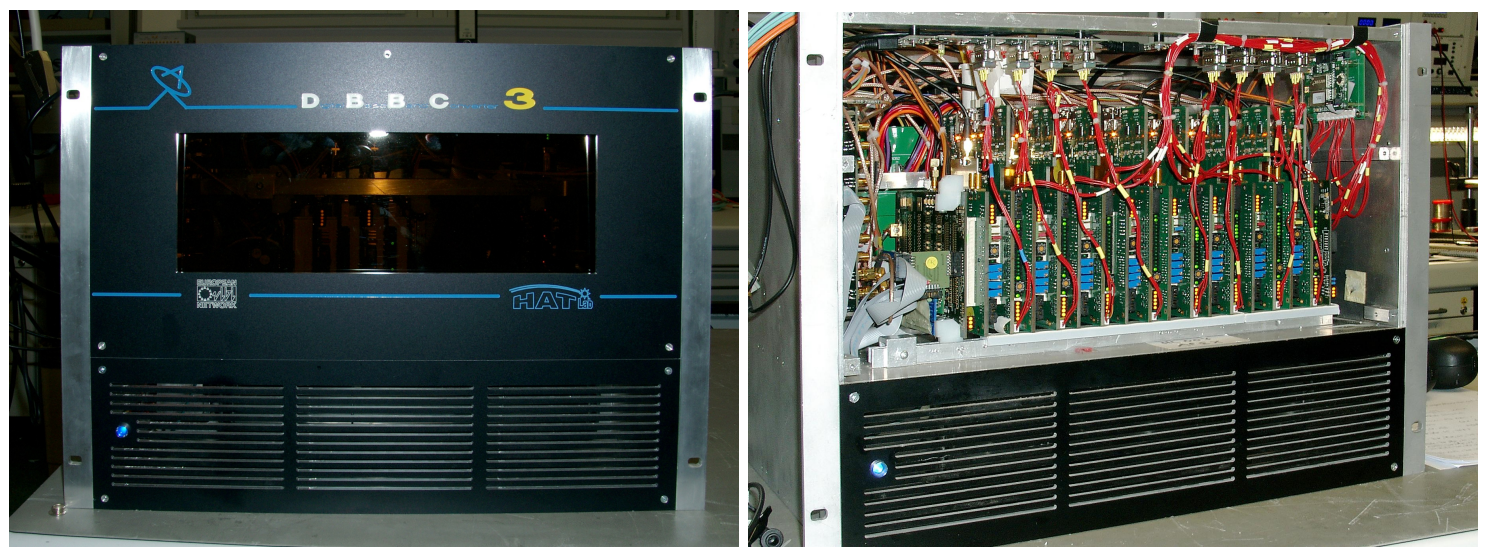

Figure 2: Left: DBBC3 front view. Right: DBBC with 8 IFs, front opened.

the receiver band is mixed down to the first Nyquist zone. This can be done with any piece of $4 \mathrm{GHz}$ taken in any position in the range of $4 \mathrm{GHz}$ to $15 \mathrm{GHz}$. The board measures and controls the power of the signal before sending it to the ADB3L sampler. In addition the GCoMo2 does gain and impedance matching at $4 \mathrm{GHz}$ to $12 \mathrm{GHz}$ before down-conversion and at $0 \mathrm{GHz}$ to $4 \mathrm{GHz}$ after down-conversion and filtering.

The ADB3L performs interleaved sampling with four sampler chips per board. The chips are e2V samplers with a bandwidth of $5 \mathrm{GHz}$ and 10-bit depth. A procedure has been designed to perform the calibration of offsets in gain and delay of the four sampler chips to prevent artefacts in the digital spectrum. For the calibration a noise source or stable noise input from a receiver can be used. The control software provides simple commands for each calibration step.

The FPGA processing board (CORE3H) with a single powerful Virtex 7 FPGA receives the sampled data and performs channelisation (see section 3) and formatting for VLBI. The data is then output as VDIF packets[1] over four optical $10 \mathrm{~Gb}$ Ethernet ports.

The DBBC3 is controlled via a command set which is similar to the $\mathrm{DBBC} 2$, but with additional functions. Each DBBC3 can have between two and eight pairs of ADB3L and CORE3H boards (see: figure 1), depending on the observing modes and bandwidth desired: two pairs for EVN, four for EHT, and six or eight for VGOS.

\section{Different observing modes available or under development}

In the last few years the firmware has been extended. The major modes are discrete sampling conversion (DSC) which simply outputs the input band as 2-bit samples, and digital down converters (DDC) which implements a set of down-converters for an input band similar to the old analogue VLBI terminals. The DDC mode is also used to realise a block-tunable polyphase filter bank (PFB). The DDC mode has been the most important one for many years in the EVN and the IVS as it provides backward compatibility with existing hardware. Standard PFB and the DSC modes require local oscillators at different stations to be tunable to identical sky frequencies.

A new mode (OCT), which allows to select arbitrary parts of the band via broad-band filters, has been developed for VGOS, EHT, and the BRAND EVN receiver [2]. This mode is meant for wide-band observations where chunks of $0.5 \mathrm{GHz}, 1 \mathrm{GHz}$, and $2 \mathrm{GHz}$ are wanted. 
Typically the same type of firmware will be loaded into each CORE3H of a DBBC for an observation. Automatic threshold calibration for 2-bit output is calculated during runtime.

The following firmwares are available:

DSC: $\quad$ whole $4 \mathrm{GHz}$ band sampled without sub-bands

DDC-L: 16 BBCs per CORE3 (U\&L) 2-4-8-16 MHz tunable within $4 \mathrm{GHz}$

DDC-V: 12 BBCs per CORE3 (U\&L) full $32 \mathrm{MHz}$ tunable within $4 \mathrm{GHz}$

VGOS: 24 bands/CORE3 $32 \mathrm{MHz}$ PFB block tunable in $4 \mathrm{GHz}$

OCT: wide-band band selection from $4 \mathrm{GHz}$

OCT1: $\quad 0-2 \mathrm{GHz}$ or $2-4 \mathrm{GHz}$

$0-1 \mathrm{GHz}, 1-2 \mathrm{GHz}, 2-3 \mathrm{GHz}$, or $3-4 \mathrm{GHz}$

$0.5-1.0 \mathrm{GHz}, 1.0-1.5 \mathrm{GHz}, \ldots$, or $3.5-4.0 \mathrm{GHz}$

OCT2: 2 filters as above per IF, output on different streams

$2 \times 2 \mathrm{GHz}$ for EHT (90\% commissioned)

A new DDC mode is currently under development. It will offer 16 base-band converters with upper and lower sidebands, tunable within $4 \mathrm{GHz}$ with bandwidths of 128-64-32-16-8-4 MHz. Also a new block-tunable PFB mode with 32 sub-bands of $128-64-32-16-8-4 \mathrm{MHz}$ is currently being developed.

\section{Outlook}

Since about 2003 the sampling rates of VLBI backends have doubled roughly every $1.6 \mathrm{y}$.

$\begin{array}{lll}2003 \text { MkIV } & 128 \mathrm{MHz} \\ 2004 & \text { DBBC1 } & 2 \times 512 \mathrm{MHz} \\ 2007 & \text { DBE } & 2 \times 512 \mathrm{MHz} \\ 2010 & \text { DBBC2 } & 2 \times 1,024 \mathrm{MHz} \\ 2014 & \text { R2DBE } & 2 \times 2,048 \mathrm{MHz} \\ 2015 & \text { DBBC3 } & 4 \times 4,096 \mathrm{MHz} \\ 2019 & \text { DBBC3H } & 4 \times 14,000 \mathrm{MHz}[2]\end{array}$

\section{References}

[1] A.R. Whitney, M. Kettenis, C. Phillips, and M. Sekido, VLBI Data Interchange Format (VDIF), in proceedings of : 8th International e-VLBI Workshop, POS (EXPReS 09$) 042$ (2009).

[2] W. Alef, G. Tuccari, S. Dornbusch, M. Wunderlich, M. Pantaleev, J. Flygare, J.D. Gallego, J.A. López-Pérez, F. Tercero, G. Schoonderbeek, J. Hargreaves, R. de Wild, L. Bezrukovs, BRAND - the next generation receiver for VLBI, in proceedings of: 14th European VLBI Network Symposium \& Users Meeting (EVN 2018), POS (EVN2018) 081 